\title{
Complicações pulmonares após cirurgia de revascularização do miocárdio
}

\author{
Pulmonary complications after myocardial revascularization surgery
}

Complicaciones pulmonares derivadas de la cirugía de revascularización miocárdica

Alana Oliveira Fagundes da Silva ${ }^{1 *}$, Fabiany Almada Costa ${ }^{2}$, Júlia Forchero Gonçalves ${ }^{3}$, Lorhainne Márjore Gomes Bastos ${ }^{4}$, Luiz Fernando Pereira Ribeiro ${ }^{5}$, Maria Luiza Cardoso Ferreira Soares ${ }^{5}$, Maysa Batista da Anunciação Vieira ${ }^{6}$, Milla Giancristofaro Dutra ${ }^{7}$, Nicolas de Vargas Franco ${ }^{8}$, Henrique Moura Parreira9.

\section{RESUMO}

Objetivo: O presente artigo tem como objetivo apresentar uma revisão de literatura acerca das principais complicações pulmonares pós-operatórias decorrentes da Cirurgia de Revascularização do Miocárdio (CRM). Revisão bibliográfica: Foram analisadas neste trabalho a importância da CRM no cenário brasileiro e mundial, bem como as principais técnicas cirúrgicas relacionadas às suas complicações pós-operatórias. Dentre essas técnicas, o emprego da circulação extracorpórea e da anestesia, bem como a dor experienciada pelos pacientes no período pós-cirúrgico, foram elencados como alguns fatores centrais responsáveis pelo desenvolvimento de problemas pulmonares. Buscou-se também compreender a fisiopatologia das condições pós-operatórias mais comuns envolvendo o aparato respiratório, como a atelectasia e o derrame pleural, para melhor analisar as medidas preventivas abordadas nesta revisão. Dentre essas medidas, empregadas para diminuir as severidades das complicações pulmonares, foi evidenciado o emprego de técnicas que exercitam e fortalecem os músculos respiratórios, visando diminuir o processo de proteólise muscular característico da CRM. Considerações finais: Entende-se que, apesar dos riscos de complicações pulmonares pósoperatórias provenientes da CRM, essa é uma terapia muito eficaz que deve ser considerada e aplicada em casos de obstrução das artérias coronárias.

Palavras-chave: Complicações pós-operatórias, Lesão pulmonar, Revascularização miocárdica.

\begin{abstract}
Objective: The present article aims to present a literature review about postoperative pulmonary complications resulting from Myocardial Revascularization Surgery (MRS). Bibliographic review: The importance of the MRS in Brazil and worldwide was analyzed in this work, as well as the main surgical techniques related to its postoperative complications. Among these techniques, both the use of the cardiopulmonary bypass and anesthesia, as well as the pain experienced by patients in the period after surgery, were described as some of the central factors responsible for the development of pulmonary disorders. It was also intended to discuss the pathophysiology of the most common postoperative conditions involving the respiratory apparatus, such as atelectasis and pleural effusion, in order to better analyze the preventive measures presented in this review. Among these measures, which are used to reduce the severity of pulmonary complications, it was presented the use of techniques of respiratory musculature training and strengthening, whose aim is to reduce the

\footnotetext{
1 Università degli Studi di Pavia (UNIPV), Pavia - IT. *E-mail: lalanafagundes93@gmail.com

2 Faculdade de Minas (FAMINAS BH), Belo Horizonte - MG.

3 Universidade Federal de Alfenas (UNIFAL), Alfenas - MG.

${ }^{4}$ Faculdade Morgana Potrich (FAMP), Mineiros - GO.

${ }^{5}$ Centro Universitário UNIFAMINAS (UNIFAMINAS), Muriaé - MG.

${ }^{6}$ Universidade Estadual do Oeste do Paraná (UNIOESTE), Cascavel - PR.

${ }^{7}$ Faculdade de Ciências Médicas e da Saúde de Juiz de Fora - Suprema (FCMS/JF), Juiz de Fora - MG.

8 Universidade do Vale do Rio dos Sinos (UNISINOS), São Leopoldo - RS.

${ }^{9}$ Centro Universitário de Caratinga (UNEC), Caratinga - MG.
} 
muscular proteolysis characteristic of MRS. Final considerations: It is understood that, in spite of the risks of postoperative pulmonary complications from MRS, this is a highly effective therapy that should be considered and applied in cases of coronary artery obstruction.

Key words: Postoperative complications, Lung injury, Myocardial revascularization.

\section{RESUMEN}

Objetivo: El presente artículo tiene como objetivo presentar una revisión de literatura sobre las complicaciones pulmonares postoperatorias derivadas de la Cirugía de Revascularización Miocárdica (CRM). Revisión bibliográfica: En este trabajo se analizó la importancia de la CRM en el escenario brasileño y mundial, así como las principales técnicas quirúrgicas relacionadas con sus complicaciones postoperatorias. Entre estas técnicas, el uso de circulación extracorpórea y anestesia, así como el dolor experimentado por los pacientes en el periodo posquirúrgico, fueron listados como algunos factores centrales responsables del desarrollo de problemas pulmonares. También se intentó comprender la fisiopatología de las condiciones postoperatorias más comunes que afectan al aparato respiratorio, como la atelectasia y el derrame pleural, para mejor analizar las medidas preventivas abordadas en esta revisión. Entre estas medidas, utilizadas para reducir la gravedad de las complicaciones pulmonares, se evidenció el uso de técnicas que ejercitan y fortalecen los músculos respiratorios, con el objetivo de reducir el proceso de proteólisis muscular característico de la CRM. Consideraciones finales: Se entiende que, a pesar de los riesgos de complicaciones pulmonares postoperatorias por CRM, esta es una terapia muy efectiva que debe ser considerada y aplicada en casos de obstrucción de las arterias coronarias.

Palabras clave: Complicaciones postoperatorias, Lesión pulmonar, Revascularización miocárdica.

\section{INTRODUÇÃO}

As doenças cardiovasculares representam um problema relacionado à saúde e à qualidade de vida, visto que são apontadas como principal causa de morte e diminuição da expectativa de vida. Estima-se que no Brasil, em 2015, as doenças coronárias foram responsáveis por mais de 250 mil hospitalizações e 16 mil mortes (LOBATO PHM, et al., 2019).

Sabe-se que as enfermidades cardíacas muitas vezes levam à realização de procedimentos cirúrgicos de alto risco, os quais são mais passíveis de desencadear complicações pós-operatórias. A cirurgia de revascularização do miocárdio (CRM) é uma cirurgia de alto risco indicada quando há a obstrução de uma ou mais artérias coronárias, situação na qual a irrigação do músculo cardíaco, posterior à obstrução, é comprometida total ou parcialmente. Esse procedimento possui o intuito de restabelecer o fluxo sanguíneo perdido ao miocárdio por meio de rotas alternativas e, caso a CRM não seja realizada, as consequências da oclusão coronária podem variar desde angina até infarto agudo do miocárdio (KNIHS NS, et al., 2017; LIMA LTA, et al., 2017).

Uma das principais técnicas utilizadas durante a revascularização miocárdica é a circulação extracorpórea (CEC), que, além de preservar a funcionalidade do aparelho cardíaco, garante um campo operatório seguro à equipe cirúrgica. No entanto, apesar desses benefícios, múltiplos sistemas, incluindo o cardiovascular e o respiratório, podem ser prejudicados pelo uso da CEC, uma vez que essa técnica foi apontada como desencadeadora de possíveis complicações associadas à resposta inflamatória sistêmica, ao aumento do tônus venoso, à lesão do miocárdio e à disfunção pulmonar (ANDRADE AYT, et al., 2019).

Além das complicações relativas à CEC, a revascularização do miocárdio, como um todo, pode desencadear complicações pós-operatórias, especialmente aquelas que envolvem o sistema respiratório, como atelectasia, edema pulmonar, pneumotórax, efusão pleural e pneumonia. De modo geral, a função pulmonar tende a ser reduzida no período imediatamente após a cirurgia por razões ainda não completamente compreendidas. Dentre essas razões, incluem-se as condições cirúrgicas, a incisão, a anestesia, a hemodinâmica do paciente, o tipo e a duração do procedimento, a drenagem pulmonar e até mesmo o nível de dor experienciada pelo paciente. Nessa perspectiva, o volume e a capacidade pulmonar tendem a diminuir e tanto a expansão torácica quanto os músculos respiratórios podem sofrer disfunções. Nota-se também uma 
relação entre o estresse cirúrgico e a desregulação do metabolismo proteico, o que induz a perda da massa muscular do paciente. O conhecimento dos mecanismos que geram esses agravos pulmonares é fundamental na busca de meios para evitá-los, destacando-se a fisioterapia nos períodos pré e pós-operatório, bem como a prevenção da proteólise muscular pós-cirúrgica (CORDEIRO ALB, et al., 2019; MENEZES TC, et al., 2018).

Diante do exposto, a presente revisão tem como objetivo explicitar, no contexto das doenças coronarianas, o papel da CRM, bem como os principais pontos de seu procedimento, tais como a circulação extracorpórea e seus impactos. Além disso, busca evidenciar a relação dessa cirurgia com as principais complicações no pós-operatório, que contemplam, principalmente, as complicações pulmonares.

\section{REVISÃO BIBLIOGRÁFICA}

As doenças cardiovasculares são consideradas um problema de atenção prioritária no Brasil e no mundo, uma vez que se destacam dentre as doenças que mais matam e diminuem a qualidade de vida. Além disso, as cardiopatias geram adversidades desde as limitações impostas ao estilo de vida dos pacientes, até o desenvolvimento de angústia, medo e insegurança, principalmente quando procedimentos cirúrgicos são necessários. Esses sentimentos geram alterações fisiológicas que podem acentuar os riscos cirúrgicos, logo, a atenção e apoio prestados aos pacientes no período pré-operatório é fundamental para diminuir tais riscos (KNIHS NS, et al., 2017). Em 2010, no Brasil, houve um crescimento notório da realização da CRM, o que melhorou a qualidade de vida dos pacientes, mas também evidenciou o quanto a capacidade pulmonar está sujeita a sofrer alterações posteriores à cirurgia, em razão da incisão e duração cirúrgica, da anestesia, das técnicas cirúrgicas empregada, além da condição hemodinâmica do paciente (CORDEIRO ALB, et al., 2019).

A CRM é a intervenção cirúrgica praticada, em sua maioria, nos casos em que as doenças ateroscleróticas não apresentam melhora. Para essas doenças, o tratamento farmacológico associado à mudança de hábitos de vida são as primeiras medidas adotadas; quando tais medidas não melhoram o prognóstico, e a possibilidade de realizar uma angioplastia também é descartada, a CRM é o procedimento de escolha. Nessa perspectiva, a obstrução do fluxo sanguíneo das artérias coronarianas desencadeia um processo de isquemia do músculo cardíaco associado à redução do lúmen vascular, resultando na diminuição da função cardiovascular devido à restrição do suprimento de oxigênio e nutrientes ao miocárdio. Conforme estabelecida a necessidade da CRM, o procedimento é iniciado pela esternotomia, seguido da aplicação de enxertos que recanalizam e restauram o suprimento sanguíneo às áreas afetadas do miocárdio. Esses enxertos são extraídos de veias e artérias do próprio paciente, sendo comumente utilizadas a veia safena e a artéria mamária interna esquerda (LIMA LTA, et al., 2017).

Apesar de haver evidências de melhores taxas de sobrevivência, a longo prazo, associadas à técnica que utiliza as duas artérias mamárias internas na CRM, sua realização é inferior quando comparada à utilização de uma única artéria mamária. Justifica-se tal fato pela maior complexidade e maior tempo cirúrgico, o que acaba desestimulando o ensino dessa técnica nas instituições acadêmicas. A partir do estudo realizado no hospital escola da Universidade de Maryland, na Austrália, comparando o emprego de ambas as técnicas entre outubro de 2012 e abril de 2015, não foram encontradas diferenças expressivas na duração das CRMs, quando realizadas por um cirurgião experiente. Já, quando realizadas por residentes, as taxas médias de morbimortalidade relacionadas ao emprego das duas artérias mamárias não foram afetadas. Sendo assim, concluiu-se seguro e vantajoso fornecer aos residentes treinamento para o uso das duas artérias mamárias internas nas CRMs (PASRIJA C, et al., 2018; HAN Z, et al., 2021).

Dentre outras técnicas utilizadas na CRM, a introdução da CEC permitiu que essa cirurgia fosse qualificada como segura e eficaz. Contudo, ao longo do tempo e à luz das possíveis morbimortalidades relacionadas à técnica, acreditava-se que complicações como a síndrome da resposta inflamatória sistêmica, isquemia miocárdica global, instabilidade hemodinâmica e alterações pulmonares, renais e neurológicas eram decorrentes da utilização da CEC. O emprego dessa técnica promove o aumento da pós-carga do ventrículo esquerdo, em decorrência da regurgitação aórtica. Essa situação desencadeia, além do aumento da pressão arterial sistêmica, o aumento da pressão atrial esquerda, que, por sua vez, tem como resultado o acúmulo de 
líquidos no interstício pulmonar, causando edema (BORGOMONI GB, et al., 2020; DIESTELMAIER K, et al., 2020).

Nos anos 90, com intuito de diminuir as complicações pós-operatórias relacionadas à utilização da CEC, surge a prática da CRM sem CEC. Em 2011, as diretrizes da American Heart Association consideraram ambas as técnicas cirúrgicas satisfatórias. Em termos de complexidade cirúrgica, a CRM sem CEC mostra-se mais desafiadora, contudo, em pacientes de alto risco, como idosos, ela pode trazer um prognóstico pós-operatório mais favorável. No Brasil, estudos realizados nos Estados de Santa Catarina e São Paulo, entre os anos de 2009 e 2016, permitiram avaliar que não houve diferenças significativas, a curto prazo, quanto à morbimortalidade de pacientes submetidos à CRM com e sem CEC. Ambos os procedimentos são equivalentes nos âmbitos segurança e eficiência, e cabe ao cirurgião avaliar qual técnica se adequa melhor às particularidades de cada caso (APOSTOLAKIS E, et al., 2017; BORGOMONI GB, et al., 2020; SANTOS MB, et al., 2018).

Os pacientes submetidos à CRM estão predispostos a desenvolver uma série de complicações, especialmente as pulmonares. Estudos recentes, realizados por meio da análise de prontuários em hospitais de Alagoas e Florianópolis, mostraram que cerca de $64 \%$ dos pacientes submetidos à CRM desenvolveram problemas pulmonares. Além disso, dentre os pacientes avaliados, foram encontrados fatores de risco que, além de serem associados ao desenvolvimento de problemas cardiovasculares, possivelmente se relacionam também com as complicações pós-cirúrgicas encontradas. Dentre esses fatores de risco estão a hipertensão arterial sistêmica, o diabetes mellitus, o tabagismo e o acidente vascular cerebral (CANI KC, et al., 2019; LIMA LTA, et al., 2017). Ademais, os casos associados à doença pulmonar obstrutiva crônica (DPOC) obtiveram maior incidência de complicações pulmonares e prolongados períodos de internação hospitalar e na UTI (CANI KC, et al., 2019).

As complicações pulmonares mais comuns à CRM são a atelectasia, derrame pleural, pneumonia, pneumotórax, embolia pulmonar e a necessidade de ventilação mecânica por mais de 24h (PAEZ RP, et al., 2019; BARBOSA JL, et al., 2019). No estudo realizado entre 2009 e 2016 com pacientes submetidos à CRM no estado de Alagoas, constatou-se que as complicações foram mais prevalentes no sexo feminino, com significância estatística, nos quadros de derrame pleural, acometendo $44 \%$ das pacientes. Além disso, 5,1\% das mulheres apresentaram pneumotórax e outros 5,1\% com insuficiência respiratória. As incidências dessas mesmas alterações nos pacientes do sexo masculino foram registradas em $21,7 \%, 0 \%$ e $0 \%$, respectivamente (LIMA LTA, et al., 2017).

As complicações respiratórias da CRM ocorrem, dentre outros fatores, principalmente pela administração conjunta da anestesia geral e de bloqueadores neuromusculares, além de alterações fisiológicas decorrentes do procedimento cirúrgico em si, que podem alterar o valor normal da relação entre ventilação e perfusão. A anestesia geral promove uma depressão no centro de controle respiratório, reduzindo os reflexos à hipóxia e à hipercapnia, além de inibir a contração do diafragma e dos músculos abdominais e torácicos. A coordenação dos músculos da faringe e do esôfago também é reduzida, principalmente quando bloqueadores neuromusculares são usados junto à anestesia (MISKOVIC A e LUMB AB, 2017).

A redução da força dos músculos respiratórios é um dos principais prejuízos pós-operatórios que podem influenciar o surgimento de diversas outras complicações pulmonares. A instabilidade torácica gerada desde a esternotomia, realizada durante a CRM, resulta em alterações significativas no processo de ventilação; além disso, outros pontos da cirurgia, como a aplicação de anestesia geral e a utilização de CEC, são fatores que também influenciam na função pulmonar. Ao término da CRM, os pacientes encontram-se predispostos ao desenvolvimento de atelectasia pulmonar e de alterações na razão entre ventilação e perfusão (CALLES ACN, et al., 2016).

O estresse cirúrgico, além de causar esse quadro de desequilíbrio do metabolismo proteico, gerando uma fraqueza mais acentuada nos músculos respiratórios em comparação à musculatura periférica, que é afetada em menor intensidade, influencia também na dor pós-operatória. Essa dor expressada pelos pacientes no pós-operatório, em grande parte devido à cicatrização da incisão cirúrgica, é outro elemento relevante às complicações pulmonares (MENEZES TC, et al., 2018). Acredita-se que as funções pulmonares possam ser 
influenciadas pela dor por até 45 dias decorridos da operação, e que a redução da expansão do tórax, responsável por uma respiração superficial, também seja induzida pela dor (CORDEIRO ALB, et al., 2019).

A atelectasia, caracterizada pelo colapso dos alvéolos pulmonares, é uma condição comum aos quadros cirúrgicos, e pode ocorrer tanto durante período intraoperatório, quanto no pós-operatório. Em cirurgias menores, essa complicação é comumente autolimitada a poucos minutos após o procedimento; já em cirurgias maiores, como a CRM, a atelectasia pode ser detectada por imagens de tomografia computadorizada por até 24 horas pós cirurgia, sendo essa marca de tempo mais comum principalmente em pacientes com comorbidades. Esse colapso alveolar ocorre por fatores como a compressão dos pulmões devido ao deslocamento cefálico do diafragma e o estreitamento das vias aéreas, decorrente da redução do volume residual pulmonar. Ademais, a utilização de altos níveis da Fração Inspirada de Oxigênio (FiO2), um dos parâmetros presentes na ventilação mecânica, relaciona-se com o desenvolvimento de atelectasia (MISKOVIC A e LUMB AB, 2017).

Outra complicação pulmonar normalmente apresentada é o derrame pleural, caracterizado pelo acúmulo de líquido na cavidade pleural. Esse quadro pode estar relacionado ao uso de drenos durante as cirurgias, o que predispõe o paciente ao desenvolvimento de lesões e ao acúmulo de secreção na região pleural, ao aumento do trabalho pulmonar durante as trocas gasosas, e à redução do volume pulmonar residual (CALLES ACN, et al., 2016).

Além disso, a presença de sedação residual reduz a contração involuntária dos músculos da faringe e do esôfago, causando descoordenação entre eles e prejudicando a desobstrução da via aérea, impedindo, por exemplo, a produção de uma tosse eficiente. Aliado a isso, têm-se os danos nas células mucociliares da traqueia, causados pela inserção do tubo durante a intubação orotraqueal, que contribuem para a obstrução das vias aéreas e a retenção de escarro (MISKOVIC A e LUMB AB, 2017; CALLES ACN, et al., 2016).

Para que complicações pós-operatórias sejam previstas a tempo de manejo, índices preditores de gravidade e mortalidade podem ser utilizados durante o período de internação dos pacientes nas UTIs. Dados coletados de pacientes submetidos à CRM entre os anos de 2011 e 2019, no Instituto Dedinje, na Sérvia, revelaram que, no que tange as complicações respiratórias, o índice que atingiu melhor sensibilidade foi o EuroSCORE, sistema europeu utilizado para avaliar riscos em cirurgias cardíacas; contudo foi o Simplified Acute Physiology Score II (SAPS II), escore simplificado que avalia a fisiologia aguda dos pacientes, que obteve maior especificidade (FRANZOTTI SAS, et al., 2019; MILUTINOVIC AV, et al., 2021).

A previsibilidade referente à readmissão hospitalar em até um mês posterior a realização da CRM pode ser analisada a partir de dados coletados durante o período pré-operatório, como etnia, uso de medicamentos como o ácido acetilsalicílico, transfusão sanguínea e presença de insuficiência renal crônica e DPOC. Esses preditores podem ser analisados com a finalidade de prover um acompanhamento médico mais rigoroso para pacientes que sejam mais propícios à readmissão pós CRM (CHIORINO CRN, et al., 2020).

Dentre as alterações fisiopatológicas posteriores à CRM, há a hipotonia dos músculos respiratórios, com consequente piora da capacidade funcional dos pulmões, devido à redução da capacidade vital e das pressões inspiratória e expiratória máximas. Esse cenário clínico pode ser avaliado por meio do TC6M, como é chamado o teste de caminhada de seis minutos. Para avaliar o dano muscular, pode-se analisar a urina de 24 horas, calculando-se a razão entre 3-metil-histidina, metabólito do catabolismo proteico muscular, e creatinina. Dessa maneira, é possível identificar a perda muscular por proteólise decorrente de hipercatabolismo, o qual é decorrente do aumento da produção de citocinas inflamatórias (IIDA Y, et al., 2016; ZANINI M, et al., 2019).

Uma estratégia para reduzir as complicações pulmonares relativas à CRM, é a aplicação do Treinamento Muscular Inspiratório (TMI), que consiste em impor resistência à inspiração, a fim de restaurar a integridade dos músculos respiratórios. O TMI ajuda na prevenção da hipotrofia e fadiga muscular, já que o fluxo sanguíneo periférico é melhorado e a capacidade funcional dos pulmões é aumentada. No TMI utiliza-se o limiar anaeróbico da variação da curva glicêmica de cada paciente, obtido por meio de repetições de incursões respiratórias em um aparelho que fornece resistência à pressão inspiratória máxima. Não obstante, o aparelho 
deve ser calibrado dentro de uma faixa de força específica para que as fibras musculares constituintes do diafragma trabalhem de modo a aumentar resistência e performance do músculo (CORDEIRO ALL, et al., 2020; BISSETT BM, et al., 2020).

\section{CONSIDERAÇÕES FINAIS}

A partir dos dados supracitados, conclui-se que a CRM é uma eficaz alternativa terapêutica para casos de obstrução coronariana, e que, sem sua realização, esses casos podem evoluir para angina e até mesmo infarto agudo do miocárdio. Uma das principais técnicas empregadas nessa cirurgia consiste na CEC, a qual garante a preservação da funcionalidade do coração e a segurança no campo operatório. Entretanto, esse é um procedimento de alto risco que pode desencadear diversas complicações operatórias e pós-operatórias, especialmente envolvendo o sistema respiratório, como atelectasia, derrame pleural, pneumonia e tromboembolismo pulmonar.

\section{REFERÊNCIAS}

1. ANDRADE AYT, et al. Complicações no pós-operatório imediato de revascularização do miocárdio. Revista Sobecc, 2019; 24(4):224-230.

2. APOSTOLAKIS E, et al. Myocardial revascularization without extracorporeal circulation; why hans't it convinced yet? Annals of Cardiac Anaesthesia, 2017; 20: 219-225.

3. BARBOSA JL, et al. Impact of Complications of Myocardial Revascularization Surgery on Expenses During Hospital Stay. Int. J. Cardiovasc. Sci., 2019; 32: 28-34.

4. BISSETT BM, et al. Which ICU patients benefit most from inspiratory muscle training? Retrospective analysis of a randomized trial. Physiother. Theory. Pract., 2020; 36(12):1316-1321.

5. BORGOMONI GB, et al. Impacto atual da circulação extracorpórea na cirurgia de revascularização miocárdica no estado de São Paulo. Sociedade brasileira de cardiologia, 2020; 115(4):595-601.

6. CALLES ACN, et al. Pulmonary complications in patients undergoing coronary artery bypass grafting at a hospital in Maceio, Brazil. Fisioter Mov, 2016; 29(4):661-667.

7. CANI KC, et al. Complicações pulmonares após cirurgia de revascularização do miocárdio: fatores associados. ASSOBRAFIR Ciência, 2019; 8(2): 41-50.

8. CHIORINO CRN, et al. Predictors of hospital readmission within 30 days after coronary artery bypass grafting: data analysis of 2,272 brazilian patients. Braz J Cardiovasc Surg, 2020; 35(6):884-890.

9. CORDEIRO ALB, et al. Behavior of pulmonary function after hospital discharge in patients submitted to myocardial revascularization. International Journal of Cardiovascular Sciences, 2019; 32(2):104-106.

10. CORDEIRO ALL, et al. Inspiratory muscle training based on anaerobic threshold on the functional capacity of patients after coronary artery bypass grafting: clinical trial. Bras J Cardiovasc Surg, 2020; 35(6):942-949.

11. DIESTELMAIER K, et al. Interdependence of VA-ECMO output, pulmonary congestion and outcome after cardiac surgery. European Journal of Internal Medicine, 2020; 81:67-70.

12. FRANZOTTI SAS, et al. Desempenho dos índices de gravidade na predição de complicações pós-operatórias de revascularização miocárdica. Arquivos Brasileiros de Cardiologia, 2020; 115(3):452-459.

13. HAN Z, et al. Application of bilateral internal mammary artery with different configurations in coronary artery bypass grafting. J Cardiothorac Surg, 2021; 16(1):1-6.

14. IIDA Y, et al. Predictors of surgery-induced muscle proteolysis in patients undergoing cardiac surgery. Journal of Cardiology, 2016; 68:536-541.

15. KNIHS NS, et al. Caminho percorrido até a cirurgia cardíaca: necessidades e expectativas no pós-operatório. Av Enferm, 2017; 35(1):30-41.

16. LIMA LTA, et al. Incidência de complicações pulmonares em pacientes submetidos a cirurgia de revascularização do miocárdio em um hospital de Maceió. Ciências Biológicas e de Saúde Unit, 2017; 4(1): 135-146.

17. LOBATO PHM, et al. Clinical course of patients undergoing myocardial revascularization surgery in a public cardiology referral hospital in Pará, Brasil. International Journal of Cardiovascular Sciences, 2019; 32(3): 217-226.

18. MENEZES TC, et al. Comparações e correlações da intensidade da dor e da força muscular periférica e respiratória no pré e pós-operatório de cirurgia cardíaca. Revista Brasileira de Terapia Intensiva, 2018; 30(4):479-486.

19. MILUTINOVIC AV, et al. Prediction value of EuroSCORE II in total arterial revascularization and its usage in the evaluation of postoperative complications: Single-center experience. Asian Cardiovasc Thorac Ann, 2021; 0(0):1-7.

20. MISKOVIC A, LUMB AB. Postoperative pulmonary complications. British Journal of Anaesthesia, 2017; 118(3):317334.

21. PAEZ RP, et al. Coronary Artery Bypass Surgery in Brazil: Analysis of the National Reality Through the BYPASS Registry. Braz. J. Cardiovasc. Surg., 2019; 34(2): 142-148.

22. PASRIJA C, et al. Bilateral internal mammary artery use can be safely taught without increasing morbidity or mortality. Ann Thorac Surg, 2018; 105:76-82.

23. SANTOS MB, et al. Comparação dos resultados iniciais entre cirurgias de revascularização do miocárdio com e sem circulação extracorpórea. Arquivos Catarinenses de Medicina, 2018; 47(2):170-181.

24. ZANINI M, et al. Associação entre consumo de oxigênio de pico e teste de caminhada de seis minutos em pacientes após cirurgia cardíaca. Fisioter. Pesqui., 2019; 26(4):407-412. 